\title{
Del socio inglés a la asociación estratégica con China: Argentina y el realismo periférico*
}

\section{From the British partner towards the strategic association with China: Argentina and peripheral realism}

\author{
Luciano Damián Bolinaga ${ }^{*}$ \\ Grupo de Estudios del Asia y el Pacífico (GEAP), Universidad Abierta \\ Interamericana, Rosario, Argentina
}

Recibido: 14 de octubre de 2014. Aprobado: 31 de mayo de 2015

\begin{abstract}
Resumen
Desde la perspectiva teórica del realismo periférico se analiza la política comercial y exterior de los países periféricos en una coyuntura de cambio internacional. Así, el periodo 1989-2014 es relevante porque se produce el cambio del epicentro económico mundial hacia el Pacífico Norte y se acelera el ascenso de China como gran potencia. El caso argentino expone como un país periférico reorienta su política comercial y exterior hacia los nuevos centros de poder internacional. Asímismo, el factor estructural sale a la luz al estudiar las relaciones bilaterales de Argentina con Inglaterra, bajo el epicentro económico del Atlántico europeo, y con Estados Unidos, ya con el eje económico sobre el Atlántico Norte. El estudio de tendencias recurrentes del sistema resulta de un alto valor instrumental para el diseño e implementación políticas comerciales y exteriores. Argentina aprovechó su vínculo bilateral y la complementación comercial con Inglaterra. Frente al ascenso de Estados Unidos optó por confrontar y tuvo que lidiar con estructuras productivas competitivas. Ante la emergencia de China como una gran potencia cabe

* El artículo presenta una síntesis del tema de investigación doctoral desarrollado con el apoyo del CONICET y en el marco de la Facultad de Ciencia Política y Relaciones Internacional de la Universidad Nacional de Rosario. Se agradece particularmente la dirección de Eduardo Oviedo y los comentarios de Carlos Escudé, quien fuera jurado del tribunal evaluador de la tesis doctoral.

** Doctor en Relaciones Internacionales (UNR, 2011) y egresado del Programa de Estudios Posdoctorales (UNTREF, 2013). Ha sido becario doctoral y posdoctoral del CONICET. Actualmente es Director del Grupo de Estudios del Asia y el Pacífico en la Universidad Abierta Interamericana (Rosario) y profesor de Historia Política Argentina en dicha institución. Es autor del libro "China y el epicentro económico del Pacífico Norte". Correo electrónico: bolinagaluciano@gmail.com
\end{abstract}


preguntarse qué tipo de vínculos comerciales y políticos mantuvo la Argentina con el país asiático.

Palabras Claves: realismo periférico, China, Argentina.

\begin{abstract}
From the theoretical perspective of peripheral realism this article is an analysis of the foreign and commercial policy of peripheral countries in a changing international environment. It is mainly focused on the 1989 - 2014 period due to the shift of the world economic axis towards the North Pacific with China rapidly rising to become a great power. From the Argentinean case, the way a periphery country readdresses its commercial and foreign policy towards the new centers of international power is analyzed. In spite of this time delimitation, the structural factor is considered when studying the bilateral relations between Argentina and England under the economic epicenter of the European Atlantic, and with United States under the economic axis of the North Atlantic. The analysis of recurring tendencies of the system provides a high instrumental value for the design and implementation of commercial and foreign policies. Argentina profited from its bilateral relation and its commercial complement with England. With the rising of the United States, Argentina chose the confrontation an also faced the competitiveness of its productive structures. As China emerges as a great power we asked: What kind of commercial and political relationship Argentina remained with this country?
\end{abstract}

Keywords: peripheral realism, China, Argentina. 


\section{Introducción}

Este artículo es una síntesis actualizada de una investigación doctoral (Bolinaga, 2011) y su desarrollo opera sobre tres niveles argumentativos. El primer nivel introduce el objeto de estudio, presenta el problema de investigación formulado, explica la metodología implementada y la hipótesis construida. El segundo, refiere a las definiciones operacionales y al marco teórico-conceptual empleado durante el análisis. Finalmente, el tercer nivel refiere a los resultados alcanzados y su validez para cotejar y convalidar esa hipótesis.

El objeto de estudio es la política comercial y exterior de los países periféricos en un contexto de cambio internacional, dado por el ascenso y descenso de grandes poderes. El caso argentino es pertinente porque explica cómo un país periférico modifica su política comercial y externa como respuesta a las tensiones provenientes del ambiente internacional. Pero no interesa cualquier tensión sino aquella que supone un recambio de las unidades que conforman el directorio de grandes potencias y, en consecuencia, una reformulación del orden internacional.

La posición de un país en la estructura de poder varía en el tiempo como consecuencia de una alteración de sus capacidades, mensuradas en términos de poder (Bolinaga, 2013b, pp. 35-36). Inglaterra fue la potencia del siglo XIX, Estados Unidos la del siglo XX y China ya rivaliza con éste el liderazgo de potencia en el siglo XXI. No hay dudas de que los países periféricos ven condicionada su política comercial y externa por el reposicionamiento de las potencias en la estructura, los registros históricos así lo verifican. Argentina sacó provecho de su vinculación con Gran Bretaña porque supo alinearse a su liderazgo internacional y porque sus estructuras productivas eran comercialmente complementarias. En contraste, frente el ascenso de Estados Unidos como gran potencia Argentina no supo adaptarse, confrontó su liderazgo internacional y, además, tuvo que lidiar con el efecto perverso de estructuras productivas competitivas y ya no complementarias, como otrora sucediera con el "socio inglés" (Bolinaga, 2011 y Escudé, 2011).

Desde finales del siglo XX la expansión de su capacidad material y su nuevo rol como gran potencia hacen que Beijing haya comenzado a ejercer mayor influencia en diferentes regiones del mundo, particularmente en África y Latinoamérica donde las estructuras productivas son complementarias de la suya. China se ha convertido en la segunda economía del mundo y compite por el primer puesto con Estados Unidos. Es el principal productor de manufacturas; el país que mayor participación tiene sobre el comercio mundial y no solo es el segundo receptor de inversión extranjera desde 2010 
sino que se ha convertido también en el tercer emisor a escala global desde 2013. La República Popular China (RPCh) es el segundo país que más invierte en defensa, por detrás de Estados Unidos; el principal acreedor de bonos de la Reserva Federal y ya desde 1971 ocupa un asiento permanente en el Consejo de Seguridad de Naciones Unidas. China irrumpió a principios del siglo XXI entre las filas de las grandes potencias, devino en un país central, lo cual favorece la relocalización del eje económico mundial sobre el Pacífico Norte y eso explica su nuevo rol tanto en el sistema productivo global como en la geopolítica internacional.

La modernización económica en China supone una transformación estructural de su capacidad productiva, la cual giró el proceso de una economía agrícola a una industrial y, al mismo tiempo, supuso la transición de una economía planificada a una de mercado; si bien este último proceso no ha concluido. La ampliación de su base material favoreció su ascenso como gran potencia y la convergencia de ambos procesos conllevó a reconfigurar sus relaciones con el resto del mundo (Oviedo, 2005; Bregolat, 2011). Y aunque la hábil retórica oficial intente encubrirlo -alegando la pertenencia del país al bloque de naciones en desarrollo- China se ha convertido en un país central, le guste o no a quien valore la cuestión. Por tanto, desde ese nuevo posicionamiento se acentúan las asimetrías con los países periféricos al tiempo que se reformulan los vínculos políticos y comerciales.

Teniendo de trasfondo el advenimiento de una nueva configuración de poder internacional, Argentina asiste a una nueva oportunidad para mejorar su inserción en el mundo que se expresa en dos interrogantes, los cuales delimitaron conjuntamente el problema de investigación: a) ¿Qué sucede con la política comercial y exterior de un país periférico cuando se reformula la composición del oligopolio de grandes poderes? y b) ¿es China un socio estratégico para la Argentina?

El marco teórico/conceptual se nutre en el "realismo periférico". Este enfoque teórico fue concebido y desarrollado por Carlos Escudé a partir del estudio de caso donde triangulaba la relación entre Gran Bretaña, Argentina y Estados Unidos. Con el objetivo de explicar el esquema de vinculación que los Estados periféricos deben mantener con las grandes potencias en pos de optimizar su inserción internacional y así contribuir al desarrollo económico propio, la maduración de sus conjeturas e inferencias teóricas/ conceptuales fueron forjando progresivamente una teoría normativa de las relaciones internacionales, como un enfoque teórico que emergió por fuera de los centros de poder mundial. Con ese propósito en el horizonte formuló tres principios para operacionalizar la política exterior basada en el realismo periférico: a) eliminar las confrontaciones po- 
líticas con las grandes potencias, reduciendo el ámbito de sus antagonismos a aquellos asuntos materiales vinculados directamente a su bienestar y a su base de poder; b) la política exterior no sólo debe calibrarse en términos de costo y beneficios sino también en función de los costos potenciales y c) se redefine el concepto de autonomía, en términos de capacidad y de costo relativo de confrontación (Escudé, 1995). Según el primer punto la confrontación solo puede ser aceptada si el desarrollo económico está comprometido o amenazado; así las malas lecturas del realismo periférico solo han contribuido al "vaciamiento y empobrecimiento" del país (Escudé, 2012, pp. 47 y 49).

Frente al surgimiento de una nueva configuración de poder donde el eje de las relaciones internacionales ha sido desplazado desde el Atlántico hacia el Pacífico resulta legítimo y vital preguntarse: ¿qué papel tiene China para la inserción internacional argentina? La potencia asiática no ejerce hegemonía en la periferia aún pero sí influencia y esto se ve muy claramente en América Latina desde 2004 al presente. El bilateralismo practicado por Beijing profundiza las asimetrías de poder con los países de la región, mientras que la modernización económica en China incrementa la demanda de materias primas. Entonces, el esquema de vinculación que se erigió tiende a reproducir las relaciones centro/periferia. Así, América Latina y Argentina -en particular- adoptaron un modelo de intercambio que favoreció las exportaciones de productos primarios a gran escala y que operó por el tradicional nexo con el capital financiero extranjero (Svampa, 2013), sólo que en esta fase toma fuerza el capital chino. Si hubiera que poner un nombre al nuevo capítulo en la historia del vínculo entre países centrales y periféricos no hay dudas, el título sería "Consenso de Beijing" (Bolinaga y Slipak, 2014).

El análisis se acentúa entre 2004 y 2014 pero resulta necesario formular dos advertencias al lector. La primera, la investigación doctoral desarrolló un segmento temporal mucho más amplio y, por lo tanto, se recomienda su lectura a efectos de lograr mayor profundización en la problemática aquí sintetizada. La segunda, a continuación se encontrará consideraciones que exceden dicha delimitación porque es necesario reconocer y dar cuenta del factor estructural en el trasfondo del objeto de estudio propuesto.

Se comprobará que el modelo de inserción internacional de un país periférico reconoce la importancia de estrechar vínculos comerciales y políticos con las nuevas potencias del oligopolio de grandes poderes. Se utilizó una metodología cuantitativa -propia de la lógica deductiva- pero para interpretar fenómenos políticos internacionales -que no pueden ser reducidos a la lógica fenoménica natural- también se implementaron técnicas de tipo cualitativas; así la lógica inductiva también estuvo presente en la investigación. 
La hipótesis construida se expresa así: Argentina reformuló su política comercial y externa como consecuencia del ascenso chino y el giro de la economía mundial hacia el Pacífico Norte. La complementación comercial facilitó la expansión del intercambio pero no aseguró la calidad del socio chino. Así, la nueva asimetría de poder cercenó el margen de negociación argentino y su capacidad para incorporar mayor valor agregado a sus exportaciones hacia el mercado chino. El vínculo establecido con China desde 2004 omitió la aplicación del realismo periférico en tanto condicionó el desarrollo económico argentino porque emergió la reprimarización productiva.

\section{Del socio inglés a la "contraparte" americana}

Los países periféricos siempre necesitan de sus vínculos con las grandes potencias para mejorar su inserción internacional: "[...] desde los países dependientes [...] la política hacia las grandes potencias siempre es relevante, por las reacciones positivas o negativas, directas o indirectas, que puede suscitar." (Escudé, 1983, p. 187). Las Provincias Unidas del Río de La Plata, desde el momento de su emancipación política, encararon un tópico tríptico (inmigrantes, capitales y comercio) el cual operó mediante su vinculación con Europa porque Inglaterra y las otras grandes potencias europeas configuraban el centro de poder mundial, por ese entonces. Inglaterra tuvo una importancia central para la política comercial y externa no sólo de Argentina sino de la mayoría de las incipientes repúblicas americanas: "Desde Waterloo hasta la Primera Guerra Mundial, el comercio internacional creció aún más rápidamente que la producción, e Inglaterra estaría en el centro de ambos desarrollos hasta que Alemania y Estados Unidos desafiaron esa posición hacia fines del periodo." (Vázquez, 1992, p. 19). Ya sea comercial o políticamente las grandes potencias ejercieron influencia en la orientación externa de las unidades periféricas del sistema internacional.

En el Río de La Plata, "Buenos Aires fue la primer provincia que se desprendió de España y la primera que se unió a Europa” (Burgin, 1969, p. 43). Londres se erigió como el eje de la política comercial y externa argentina, primero por su posicionamiento internacional y, luego, por la complementación comercial entre ambos países. La politización de los rurales preparó el terreno para cimentar el modelo agroexportador y de la mano de ese esquema de vinculación se coronó el "socio inglés".

Existe un amplio consenso académico acerca de la afiliación y dependencia argentina a la esfera británica. Vázquez Presedo (1992) utilizó la expresión "auge del sistema 
con centro en Londres" para explicar la coyuntura internacional en el cual Argentina fue diseñando su modelo de inserción durante el siglo XIX. Más aún, ese consenso académico cobra sentido a través de una serie de conceptos: la "dependencia nacional" (Puig, 1984), el "desarrollo dependiente de la economía británica" (Escudé, 1983) y la "afiliación a la esfera de influencia británica" (Gosende, 2007).

La etapa agroexportadora mejoró la vinculación del país con las grandes potencias y su inserción en la económica mundial. Entre 1880 y 1912, Argentina exportaba a Inglaterra más del 30\% de su producción y de sus importaciones totales el $40 \%$ provenía del mercado británico (Rapoport, 2000, p. 30). La inversión total que recibió Argentina pasó de 30 millones de libras en 1875 a más de 600 millones en 1914, siendo el 60\% de origen británico (Vázquez, 1992, pp. 34-35). ¿Pero cuánto de esa relación generó desarrollo económico para el pueblo? Hubo un sector de la sociedad, la oligarquía terrateniente, que sacó mayores beneficios que el resto. El modelo facilitó la concentración de tierras e imposibilitó una colonización de pequeños productores al estilo norteamericano. Hasta las concesiones en materia de ferrocarril actuaron en desmedro del pueblo, lo importante era conectar los factores productivos con el puerto de Buenos Aires y no las ciudades, los pueblos o las personas. Los caminos de hierro originaron un "país abanico".

Entre 1913 y 1933 hubo acontecimientos que socavaron la posición del "socio inglés". El aumento de la tasa de interés en Londres repatrió capitales y así la inversión británica en Argentina cayó. En 1914 Estados Unidos desplazó a Inglaterra como principal proveedor del país y eso abrió una relación triangular donde el superávit comercial con Londres financiaba el déficit con Washington. Las inversiones norteamericanas en el país comenzaron a aumentar sistemáticamente y a rivalizar con las británicas. Al respecto:

La mayor parte de los capitales que ingresaron al país después de 1923 tuvieron su origen en los Estados Unidos. La inversión total norteamericana en la Argentina creció de unos 75 millones de pesos oro (el peso oro era equivalente al dólar de la época) a más de 800 millones, entre 1920 y 1931 [...] El National City Bank, que había abierto por aquel entonces en Buenos Aires la primera sucursal exterior de un banco norteamericano, decidió prestar 15 millones de dólares, y así fue como comenzó nuestra reorientación hacia el mercado monetario de Nueva York. (Vázquez, 1992, p. 86)

Argentina no supo o sus gobiernos no quisieron reorientar la política comercial y exterior sobre la lógica de la nueva configuración de poder que emergió de la Primera Guerra Mundial. Más aún, en el contexto de la Gran Depresión, Argentina renovó su apuesta al nexo entre el modelo agroexportador y el socio inglés. Así, se firmó uno de los 
acuerdos más polémicos en la historia argentina, el cual dio lugar a los grandes negociados de los frigoríficos británicos, el Pacto Roca-Runciman de 1933.

A diferencia de lo que había acontecido con Inglaterra, la vinculación con los Estados Unidos osciló permanentemente entre la discordia y la cooperación, sin poder estabilizarse ni generar continuidad en materia de política comercial o exterior. Argentina no sacó provecho del ciclo expansivo norteamericano sino que, por el contrario, sufrió el costo de confrontar con la potencia en ascenso. Y si bien esto se remonta en el tiempo hasta las posiciones antagónicas en torno al panamericanismo, es en el siglo XX cuando cobró más fuerza. Así, entre 1914 y 1947, Argentina mantuvo una política comercial y exterior disfuncional a su inserción internacional, aunque no cabe duda que esa posición respondiera a ciertos intereses de un sector específico de la clase política. Como sea, la errática percepción argentina acerca del cambio internacional ocurrido contribuyó a originar -junto con otras causas- un proceso de desinserción que afectó su posicionamiento internacional. Entre 1942 y 1947 la Casa Blanca hostigó deliberadamente al país al punto de aislarlo diplomática y comercialmente.

Ya hacia finales de la década del 40, hubo un claro acercamiento de Argentina hacia Estados Unidos cuya génesis remite al reconocimiento del liderazgo económico y financiero internacional de dicho país por los sucesivos gobiernos argentinos, desde Perón hasta Menem. Asimismo, ese reconocimiento no alcanzó para generar una cooperación estable con la Casa Blanca. Antagonismos y divergencias continuaban latentes en la relación bilateral y eso desalentaba y discontinuaba los impulsos tendientes a alinear al país con Washington. A la desconfianza política se sumaban las rupturas constitucionales (1955, 1966 y 1976) que, en mayor o menor medida, también condicionaban el vínculo bilateral.

En todo caso, siguiendo la línea analítica de Puig (1984), no había duda que el centro de referencia en la política exterior argentina pasaba a ser Estados Unidos, pero no en un contexto de dependencia sino de autonomía. Mientras que la "dependencia nacional con referencia a Gran Bretaña” era congruente con la confrontación hacia Estados Unidos y el aislamiento respecto de América Latina, el nuevo esquema de autonomía heterodoxa suponía armonizar la relación política y diplomática con Estados Unidos e incrementar la interacción política y comercial en el sistema regional. "La Argentina ha sido siempre un país que vivió mirando a Europa, pero en este periodo Europa pasa a un segundo plano [...]" Gosende, 2007, p. 153. Ya para 1962 Estados Unidos se presentaba como el principal acreedor de deuda argentina, 85 millones de los 284 millones registrados en ese entonces respondían al Tesoro norteamericano (Escudé y Cisneros, 2000). 
Entonces, al tiempo que se cerraban los mercados europeos, los americanos y asiáticos fueron cobrando mayor importancia (ver Tabla 1).

Finalmente, tras más de un siglo de idas y vueltas donde los constantes antagonismos impidieron generar una política comercial y exterior estable con Estados Unidos, la alineación política se logró bajo los gobiernos de Carlos Menem, entre 1989 y 1999. La reformulación del vínculo permitió destrabar negociaciones comerciales y financieras e incentivar la inserción comercial del país en el mundo. Más aún, Argentina se convirtió en un aliado de la Organización del Tratado del Atlántico Norte (OTAN). Pero la internalización del Consenso de Washington dejó poco margen para un modelo de desarrollo económico exitoso, en tanto preparó el terreno para el vaciamiento del Estado y la expansión de la pobreza. Con lo cual, se puso al descubierto que poco tenía que ver esa alineación política con Estados Unidos con la esencia del realismo periférico; aun cuando su creador hubiera ocupado un lugar importante como asesor del entonces canciller Guido Di Tella. Es decir, una vez más se comprometió el interés -definido en términos de desarrollo económico- en pos de evitar una confrontación política con una gran potencia. De ahí la mala lectura que se hizo del realismo periférico, en efecto, la única confrontación que un país periférico debe tener con una gran potencia es cuando su desarrollo económico está comprometido. Brasil comprendió esto mucho antes que Argentina y su esquema de vinculación comercial y político con Estados Unidos se basó en esa aceptación. El resultado fue una mejora en el posicionamiento internacional de Brasil que le permite hoy estar dentro del directorio de grandes potencias (Bolinaga, 2013b, p. 199).

Hay que destacar que aún pese a la competitividad entre estructuras productivas, los impulsos de alineación de Argentina con Estados Unidos, permitieron aumentar las exportaciones a dicho mercado. No obstante, pronto harían su entrada en escena dos nuevos socios que superarían la importancia comercial relativa de Estados Unidos. Por un lado, Brasil que de la mano del proceso de integración regional se configuró como el principal socio de Argentina. Por otro lado, China se posicionó como el segundo socio comercial de Argentina dada su modernización económica y su ascenso como gran potencia. Más adelante se mostrará la relación de Argentina con sus socios comerciales comparativamente (ver Tabla 2).

Coetáneamente con la configuración de los nuevos socios comerciales para la Argentina, el proyecto norteamericano de configurar una Zona de Libre Comercio desde Alaska hasta Tierra del Fuego naufragó ante la IV Cumbre de las Américas en Mar del Plata, en noviembre de 2005. "El gobierno argentino, además de anfitrión, fue uno de los artífices del fracaso de ese proyecto estadounidense." (Morgenfeld, 2011, p. 421). Pero 
nueve años más tarde, el peso de Estados Unidos vuelve a caer sobre la Argentina en torno a la cuestión de los "fondos buitres". Pocas veces se han visto tantas idas y vueltas, tantas contradicciones y tantos intentos discontinuados de acercarse a una gran potencia. Tanto la fricción política como la competitividad comercial han truncado sistemáticamente todos los intentos de cooperación.

Argentina concedió siempre una importancia vital a sus vínculos con todas las naciones del mundo dada su vocación pacifista pero incluso con anterioridad a su formación como Estado-nación ya daba prioridad a las relaciones con las potencias. Pero el vínculo con las potencias no define el modelo de desarrollo económico del país, por el contrario, este debe ser anterior y, en consecuencia, la política comercial y externa se vuelven instrumentos puestos a su servicio. ¿Cómo saber si vale a la pena confrontar o no con una potencia sin haber definido el esquema de desarrollo económico? Desde la lectura del realismo periférico, sería imposible.

En rigor, Argentina sacó más provecho de su vínculo con las potencias con las cuales tuvo complementación comercial. No obstante, esto no garantizó su desarrollo económico. Ante el ascenso de China la relación comercial y política con Argentina ganó densidad. La pregunta que resta formular es: ¿podrá el "socio chino" atenuar los efectos perversos del constante antagonismo con Estados Unidos?

\section{La reorientación comercial argentina (1960-2014)}

La relocalización de las actividades productivas y la descomposición del proceso industrial, lógicas propias de la acumulación capitalista, conllevan a cambios en la ruta del comercio internacional que determinan el intercambio entre los países. Además, los procesos de ascenso y descenso de grandes poderes suelen implicar que el país en declive demande menos productos, mientras que aquel en auge tiende a incrementar sustancialmente su demanda. Por supuesto, los productos que más se destacan -dada la necesidad de mantener el proceso de modernización económica- son los productos primarios, de ahí que en el corto plazo tiendan a mejorarse los precios internacionales de los mismos. Las potencias en ascenso buscan asegurar el acceso a los recursos naturales necesarios para garantizar su modernización, así "El consumo de materias primas varía dependiendo de la 'madurez económica' de los Estados” (Renouvin y Duroselle, 2001, p. 139). Desde las relaciones internacionales la importancia de controlar las materias primas ha brindado siempre explicaciones a la expansión territorial y a la extensión de influencia 
informal de las grandes potencias (Keohane, 2005, p. 32). Ante el cambio del epicentro económico hacia el Pacífico y el ascenso de China, es de suponer que se reorientó el comercio internacional y mejoró el nivel de precios internacionales de los productos primarios (por ejemplo, aluminio, cobre, soja, entre otros).

$\mathrm{Al}$ analizar el caso argentino, ver tabla 1, se observa que la vinculación comercial entre 1960 y 2014 sufrió importantes cambios. En 1960 las exportaciones argentinas a Europa eran el 70\% del total exportado, mientras que América representó un 25\% y Asia sólo un 5\%, aproximadamente. No obstante, coetáneamente al proceso de integración europeo las ventas a esa región cayeron de manera estructural: 59\% en 1970; 54\% en 1980; $38 \%$ en 1990; 19\% en el 2000 y tan solo un 17\% en 2014.

Tabla 1

Comercio argentino por continentes, 1960/2014 (en porcentaje)

\begin{tabular}{lccccccc}
\hline \multicolumn{7}{c}{ Exportaciones Argentinas } \\
\hline Región & 1960 & 1970 & 1980 & 1990 & 2000 & 2010 & 2014 \\
África & 0,50 & 1,05 & 2,73 & 3,36 & 4,07 & 5,87 & 7,05 \\
América & 24,88 & 30,40 & 33,96 & 42,03 & 61,21 & 49,42 & 47,15 \\
Asia & 4,84 & 8,75 & 8,90 & 15,69 & 13,45 & 23,03 & 25,32 \\
Europa & 69,60 & 59,67 & 54,30 & 38,33 & 19,02 & 19,42 & 17,02 \\
Oceanía & 0,04 & 0,10 & 0,09 & 0,18 & 0,49 & 0,5 & 1,02 \\
Indeterminado & 0,14 & 0,03 & 0,02 & 0,59 & 1,76 & 1,76 & 2,01 \\
\hline & & Importaciones Argentinas & & & \\
\hline Región & 1960 & 1970 & 1980 & 1990 & 2000 & 2010 & 2014 \\
África & 1,12 & 0,64 & 1,39 & 0,50 & 1,75 & 0,88 & 1,42 \\
América & 21,45 & 50,53 & 44,98 & 57,02 & 54,82 & 52,55 & 49,02 \\
Asia & 6,08 & 8,14 & 17,31 & 8,6 & 15,87 & 23,60 & 26,30 \\
Europa & 69,10 & 40,42 & 35,39 & 32,46 & 26,57 & 21,56 & 21,04 \\
Oceanía & 0,32 & 0,11 & 0,88 & 1,40 & 0,34 & 0,57 & 0,31 \\
Indeterminado & 1,93 & 0,16 & 0,05 & 0,02 & 0,65 & 0,84 & 1,01 \\
\hline
\end{tabular}

Fuente: Elaboración propia en base a estadísticas del INDEC (Cuadernos de Comercio Exterior 1964-1995 e Indec Informa 2000-2014). 
Simultáneamente, la concreción del Mercado Común del Cono Sur (MERCOSUR) y, particularmente, el eje argentino-brasileño estimuló el comercio intrarregional con lo cual América incrementó su participación en las ventas argentinas de forma progresiva y sustancial: $25 \%$ en $1960 ; 30 \%$ en $1970 ; 34 \%$ en $1980 ; 43 \%$ en $1990 ; 61 \%$ en el 2000 y $47 \%$ en 2014. De igual forma, la expansión de China y la reorientación del comercio mundial hacia el Pacífico también estimuló el comercio con Asia: entre 1970 y 1980 supo representar el 9\%; para 1990 llegó al 16\%, cayó al 14\% en el 2000 -merma que se explica por la crisis asiática- y alcanzó un 26\% en 2014.

En 2007 las exportaciones argentinas al Asia superaron, por primera vez en la historia del país, a las destinadas a Europa según los datos registrados por el INDEC. Más aún, esta tendencia reapareció con más fuerza en el 2010 y desde entonces se ha mantenido constante hasta nuestros días.

La tabla 1 también da cuenta del cambio estructural en materia de importaciones. América y Asia han desplazado sistemáticamente a Europa como proveedores de manufacturas y bienes de capital. Así, Europa redujo progresivamente su participación en las importaciones argentinas: cayó del $69 \%$ en 1960 al 21\% en 2014. Mientras tanto, América y Asia pasaron de $21 \%$ a $49 \%$ y de $6 \%$ a 26\%, respectivamente en igual periodo de tiempo.

Entonces, es claro que los mercados americanos y asiáticos han sido más dinámicos para la inserción comercial argentina. No obstante, una tendencia que no debe omitirse es la caída del comercio intrarregional durante la primer década del siglo XXI. Así, parte de la expansión del comercio con Asia se explica por la caída del comercio intrarregional. Más aún, detrás de la caída del comercio intrarregional está puntualmente el fenómeno chino, es decir, Beijing viene ganando cuota del mercado argentino y desplaza a tradicionales proveedores de manufacturas de alto y bajo contenido tecnológico (Slipak, 2014). Brasil está siendo desplazado por China, entre 2013 y 2014 las importaciones argentinas de origen brasileño cayeron 4.824 millones de dólares. Indudablemente, la expansión de los vínculos comerciales de Argentina con China -en estos términos- está poniendo en jaque al MERCOSUR. Pero Argentina no es la única que apuesta al socio chino, también lo hacen los otros miembros del bloque, lo que no hay es una política común desde el MERCOSUR que revierta la asimetría de poder que supone negociar bilateralmente con Beijing.

En materia de exportaciones argentinas al mercado chino se puso al descubierto una tendencia hacia la reprimarización productiva. Es decir, Argentina perdió capacidad 
para incorporar mayor valor agregado y, en consecuencia, se incrementó progresivamente la venta de productos primarios.

Si bien definir el concepto de reprimarización no es parte del objetivo de este artículo, se presenta como un término vital para comprender las fases de una estructura productiva. La primarización supone que la producción está concentrada en productos primarios. La desprimarización supone la desconcentración de la producción primaria y la incipiente producción de manufacturas industriales. La industrialización, por su parte, se contrapone a la primarización y supone una producción concentrada en manufacturas industriales. La reprimarización, finalmente, supone el paso de una fase de industrialización o desprimarización a una nueva instancia de primarización. Al respecto de estos cambios en la estructura productiva de un país, la modernización económica ha sido asociada tradicionalmente con los procesos de desprimarización e industrialización, nunca con economías primarias.

Slipak (2013) se pregunta “¿De qué hablamos cuando hablamos de reprimarización?" con la intención de exponer las diferentes aceptaciones que supone el concepto que no se limita únicamente a la problemática del valor agregado sino que supone cambios estructurales de gran envergadura para las economías. En este artículo se adhiere a la lógica argumentativa de Slipak aunque se enfatiza la problemática que gira en torno a la posibilidad de incorporar mayor valor agregado. Al respecto del comercio entre América Latina y China, recientes estudios no sólo sostienen la reprimarización sino que, además, incorporan el componente extractivista: "Debido a su alta intensidad en productos de las industrias extractivas, las exportaciones de América Latina a China generan relativamente menos empleo y un mayor impacto ambiental que los envíos al resto del mundo." (Comisión Económica para América Latina y el Caribe, 2015, p. 48).

El cambio estructural acontecido en la orientación comercial de Argentina también implicó ajustes de su política exterior. Hubo una transferencia de mayores recursos (financieros y de personal profesional). Cabe pensar en la apertura de nuevas oficinas consulares, centros de promoción y secretarias agrícolas. ¿Se trata de una nueva estratégica en materia de política comercial y exterior hacia el Asia? Se podría decir que sí, pero no hacia el Asia en general sino particularmente hacia China. No obstante, la lógica de una estrategia supone una posición proactiva y la política exterior argentina hacia la RPCh ha sido meramente reactiva desde 2004. Cabe pensar que si la estrategia argentina hubiera sido general hacia el Asia, esta región hubiera incrementado aún más su relevancia para la inserción comercial del país. 
Argentina -que aún sigue siendo un país periférico- debe hacer uso con la mayor eficacia posible de los recursos con los que cuenta para mejorar su inserción comercial internacional, lo cual supone al menos tres procesos: a) diversificar y expandir sus exportaciones; b) aumentar progresivamente el valor agregado de sus exportaciones (desprimarizar e industrializar) y c) asegurar el acceso a bienes de capital. De estos tres procesos el socio chino obstaculizó al menos los dos primeros.

Tabla 2

Exportaciones argentinas a Inglaterra, EUA, Brasil y China, por porcentaje (1960-2014)

\begin{tabular}{ccccccccc}
\cline { 2 - 8 } & \multicolumn{2}{c}{ Inglaterra } & \multicolumn{2}{c}{ Estados Unidos } & \multicolumn{2}{c}{ Brasil } & \multicolumn{2}{c}{ China } \\
\cline { 2 - 8 } & $\begin{array}{c}\text { \% sobre } \\
\text { X Totales }\end{array}$ & $\begin{array}{c}\text { Posición } \\
\text { como } \\
\text { socio }\end{array}$ & $\begin{array}{c}\% \\
\text { sobre X } \\
\text { Totales }\end{array}$ & $\begin{array}{c}\text { Posición } \\
\text { como } \\
\text { socio }\end{array}$ & $\begin{array}{c}\text { \% } \\
\text { sobre X } \\
\text { Totales }\end{array}$ & $\begin{array}{c}\text { Posición } \\
\text { como } \\
\text { socio }\end{array}$ & $\begin{array}{c}\% \\
\text { sobre X } \\
\text { Totales }\end{array}$ & $\begin{array}{c}\text { Posición } \\
\text { como socio }\end{array}$ \\
\hline 1960 & 20,5 & $1^{\circ}$ & 8,4 & $4^{\circ}$ & 7,7 & $5^{\circ}$ & 0,1 & N/S \\
1970 & 5,4 & $4^{\circ}$ & 8,9 & $2^{\circ}$ & 7,8 & $4^{\circ}$ & 0,1 & N/S \\
1980 & 2,5 & $7^{\circ}$ & 8,9 & $3^{\circ}$ & 9,5 & $1^{\circ}$ & 2,3 & N/S \\
1990 & 1,5 & $15^{\circ}$ & 13,7 & $1^{\circ}$ & 11,5 & $2^{\circ}$ & 1,9 & $14^{\circ}$ \\
1995 & 1,4 & $13^{\circ}$ & 7,3 & $2^{\circ}$ & 26,2 & $1^{\circ}$ & 1,3 & $17^{\circ}$ \\
2000 & 0,9 & $24^{\circ}$ & 11,9 & $2^{\circ}$ & 26,5 & $1^{\circ}$ & 3,3 & $6^{\circ}$ \\
2005 & 0,9 & $21^{\circ}$ & 11,3 & $3^{\circ}$ & 15,7 & $1^{\circ}$ & 8,2 & $4^{\circ}$ \\
2010 & 1,1 & $25^{\circ}$ & 5,1 & $4^{\circ}$ & 21,2 & $1^{\circ}$ & 8,5 & $2^{\circ}$ \\
2014 & 1,2 & $21^{\circ}$ & 6,2 & $3^{\circ}$ & 21,3 & $1^{\circ}$ & 6,4 & $2^{\circ}$ \\
\hline
\end{tabular}

Fuente: Elaboración propia en base a estadísticas del INDEC (Cuadernos de Comercio Exterior 1960/1995 e INDEC Informa 2000/2014).

La tabla 2 matiza el cambio estructural en materia de socios comerciales. Inglaterra representó el 20\% de las exportaciones y fue el principal destino en 1960, no obstante, fue perdiendo importancia de forma progresiva. Así, otros países europeos la desplazaron como socio relevante, por ejemplo: Italia, Holanda y España. Esto provocó que Inglaterra pasara del primer puesto en 1960 al décimo quinto en 1990 y, más aún, al vigésimo primero en 2014. Por otro lado, Estados Unidos representó entre 1960 y 1990 un 9\% del total exportado, llegó al 14\% en 1990 y si bien mantuvo su relevancia entre 2000 y 2005 (por arriba del $10 \%$ del total de las ventas argentinas), desde entonces fue decreciendo hasta representar el $6 \%$ en el 2014. A pesar de esto último, Estados Unidos ha logrado mantener su importancia 
relativa como socio comercial de Argentina porque ha estado dentro de los principales cuatro destinos de sus exportaciones, en dicho período. Cabe destacar que al fortalecerse el comercio intrarregional el intercambio con países limítrofes también tomó fuerza, así en algunos años el mercado chileno fue más importante que el norteamericano, por ejemplo.

Como se viene explicando, Brasil y China fueron ganando peso en el esquema comercial argentino y fueron los dos mercados más importantes para explicar la expansión de las exportaciones argentinas en América y Asia. Brasil pasó de ser el quinto destino en 1960 al segundo en 1990 y entre 1995 y 2014 se posicionó, indiscutidamente, como el principal socio comercial de la Argentina: representando más del 20\% de las exportaciones totales. China, por su parte, en términos relativos tuvo una importancia menor entre 1990 y 1995 pero desde el 2000 en adelante fue mejorando su posición. Pasó del sexto puesto en 2000 al segundo en 2010, posición que mantiene aún hoy. China ha representado entre 2010 y 2014, entre el 6\% y el 8\% del total de las exportaciones argentinas.

Tabla 3

Esquema comercial argentino en el 2014

\begin{tabular}{llll}
\hline Destinos de export. & Origen de import. & Déficits & Superávits \\
\hline Brasil, 14.926.335 & Brasil, 14.336.549 & China, 6.111.434 & Chile, 2.187.271 \\
$(21,3 \%)$ & $(21,9 \%)$ & EUA, 4.860.788 & Venezuela, 2.098.371 \\
China, 4.650.042 & China, 10.761.477 & Alemania, 1.916.330 & Argelia, 1.654.004 \\
$\mathbf{( 6 , 4 \% )}$ & $(\mathbf{1 6 , 4 \% )}$ & Bolivia, 1.364.553 & Canadá, 1.176.432 \\
EUA, 4.528.546(6,2\%) & EUA, 8.907.453 & Francia, 1.215.932 & Uruguay, 1.176.426 \\
Chile, 3.010.267 (4,1\%) & $(13,6 \%)$ & & \\
Venezuela, 2.108.045 & Alemania, 3.536.651 & & \\
$(2,9 \%)$ & $(5,4 \%)$ & & \\
& México, 1.645.915 & & \\
& $(2,5 \%)$ & & \\
\hline
\end{tabular}

Unidad monetaria: miles de USD.

Fuente: Elaboración propia en base a estadísticas del INDEC (Indec Informa, junio, 2014).

La tabla 3 muestra el esquema comercial argentino en el 2014. Brasil y China, ocuparon el primer y segundo puesto como proveedores del país y como destino de sus exportaciones, en tanto el tercer puesto lo ocupó Estados Unidos. En materia de principales destinos de las exportaciones los países europeos fueron desplazados, en su mayoría por países limítrofes. En 2014, Bolivia desplazó a Holanda que había sido el único que se destacaba en 2013. En materia de importaciones, Alemania se muestra como el país europeo 
más relevante para las importaciones de manufacturas y bienes de capital, con lo cual se posiciona en la cuarta posición del ranking. Hoy no quedan vestigios de lo que alguna vez fue el socio inglés. En materia de déficits comerciales llama la atención que Argentina no ha logrado tener saldo positivo con las grandes potencias, es decir, los tres déficits más importantes que tuvo el país en 2014 fueron con China, Estados Unidos y Alemania. En contraste, los superávits comerciales más importantes que recibió provienen de países periféricos: Chile, Venezuela, Argelia, Perú y Uruguay.

Es evidente que Argentina tiene gran dependencia de las grandes potencias para colocar sus productos y abastecerse de bienes de capital, no obstante, en el esquema global mantiene desequilibrios comerciales con la mayoría de ellas.

\section{Argentina ante el ascenso de China como gran potencia}

China viene en expansión desde 1943 pero su fase de acenso recién se aceleró entre 1989 y nuestros días. En este sentido, hay que destacar que la relación bilateral entre Argentina y China fue tomando fuerza por los impulsos que se derivaron del ascenso chino, lo cual va dando la pauta de esa política reactiva recién mencionada. A groso modo, se pueden distinguir tres grandes etapas (ver Tabla 4).

Tabla 4

Etapas en la relación sino-argentina desde 1945

\begin{tabular}{|c|c|c|}
\hline Primera Etapa (1945-1972) & Segunda Etapa (1972-2004) & Tercera Etapa $(2004-\ldots)$ \\
\hline $\begin{array}{l}\text { Establecimiento de las } \\
\text { relaciones diplomáticas } \\
\text { con la República de } \\
\text { China. Poca relevancia } \\
\text { política y comercial, } \\
\text { salvo determinados picos } \\
\text { comerciales. Relaciones } \\
\text { entre países semi- } \\
\text { periféricos. }\end{array}$ & $\begin{array}{l}\text { Normalización de las } \\
\text { relaciones diplomáticas } \\
\text { con la República Popular } \\
\text { China. Incremento de la } \\
\text { relevancia política y aumento } \\
\text { del intercambio comercial. } \\
\text { Apertura de nuevas } \\
\text { representaciones. Relaciones } \\
\text { entre países semi-periféricos. }\end{array}$ & $\begin{array}{l}\text { Gran relevancia política y } \\
\text { comercial. Se forma una asociación } \\
\text { estratégica (2004) y posteriormente } \\
\text { se eleva al rango integral (2014). } \\
\text { En el intercambio comercial } \\
\text { hay reprimarización productiva } \\
\text { y creciente extractivismo. Las } \\
\text { relaciones comerciales y políticas } \\
\text { se reformulan en términos centro- } \\
\text { periferia. }\end{array}$ \\
\hline
\end{tabular}

Fuente: Elaboración propia. 
¿Por qué Argentina buscó establecer relaciones diplomáticas con China en 1945 y no antes con el "Imperio del Centro" durante el siglo XIX o con la República nacida en 1911? El motivo fue explicado por el canciller argentino, César Ameghino, en diálogo con su amigo el doctor José Arce quien sería designado como primer embajador en el país asiático: "China tendrá asiento permanente en el Consejo de Seguridad y juntamente con los otros cuatro grandes poderes reconocidos [...] dispondrá de un voto privilegiado. Nos hemos incorporado a la referida organización y necesitamos hacernos del mayor número de amigos." (Arce, 1958, p. 15). De modo que, en la primera etapa el impulso se explicó por el lugar que China fue llamada a ocupar en Naciones Unidas.

El 1 junio de 1945 se establecen relaciones diplomáticas con la República de China. Hay que destacar, concordando con Oviedo (2010), que si bien el proceso se da en forma tardía -sí se comparara con otras naciones latinoamericanas- Argentina nunca forzó a China a firmar "tratados desiguales" durante su ciclo de contracción y, de hecho, tomó distancia de las prácticas colonialistas de las grandes potencias. Por eso, la relación bilateral no registra obstáculo histórico alguno que pudiera opacar el vínculo de amistad entre ambos pueblos.

En el contexto de la Guerra Fría y de la Revolución Comunista de Mao, el país se vio obligado a tomar una posición concreta que se plasmó en la decisión de mantener el vínculo diplomático con la República de China, un gobierno pro-occidental. Esta cuestión fue un límite concreto a la Tercera Posición de Perón y desde entonces Argentina reconoció a la República instalada en Taiwán como única representante del pueblo chino. Esto se mantuvo hasta llegar la década del 70, momento en el que se produce el segundo impulso para la relación bilateral y que generó el paso a la segunda etapa.

El cambio de representación china en el Consejo de Seguridad de las Naciones Unidas y el acercamiento entre Nixon y Mao implicaron: "La reincorporación de China al juego diplomático mundial y el aumento de las opciones estratégicas para Estados Unidos [...]" (Kissinger, 2012, p. 289). Pero sin lugar a dudas, para Beijing también porque culminaba su década de aislamiento internacional. Así, el resto de los países alineados con Occidente fue normalizando sus relaciones con Beijing y Argentina no fue una excepción.

El giro de la posición política del gobierno de Washington frente al tema de las dos Chinas indujo al canciller Luis María de Pablo Pardo a proponer al gobierno argentino dar los pasos necesarios para reconocer a la China Comunista. (Lanús, 2000, p. 91) 
La negociación bilateral se inició por medio de la representación argentina en Bucarest (desde septiembre de 1971) y, paralelamente, se desarrolló la cuestión del cambio de representación de China en el Consejo de Seguridad. Argentina votó a favor de interpretar la cuestión como importante, pero al votar la Resolución 2758 se abstuvo por considerar que no era coherente con el hecho de que aún mantenía relaciones con Taiwán. Finalmente, la normalización de relaciones diplomáticas entre la Argentina y China continental verificó una discontinuidad de la política exterior argentina desplegada a favor de Taipei (entre 1949 y 1972) y, su máxima expresión llegó de la mano del "Comunicado Conjunto de Bucarest", el 16 de febrero de 1972. Los elementos centrales del documento son: a) ambos gobiernos deciden "normalizar relaciones diplomáticas"; b) se reconoce los principios de soberanía, integridad territorial, no intervención, igualdad y beneficio mutuo; c) Argentina reconoce al gobierno de la RPCh como único representante legal de China y toma nota de su reclamo sobre el territorio de Taiwán; d) China reconoce la jurisdicción de Argentina sobre la zona marítima adyacente a sus costas dentro del límite de 200 millas náuticas; e) ambos gobiernos acordaron "medidas eficaces" para el desarrollo de las relaciones comerciales y f) intercambio de Embajadores tan pronto como sea posible.

La decisión de normalizar relaciones con Beijing -distinta a la de "establecer relaciones"- dejó al descubierto un cambio en la orientación externa argentina. Se clausuró la embajada en Taipei por medio del Decreto $\mathrm{N}^{\circ} 4.199$ (4 de julio de 1972) y se designó al doctor Eduardo Bradley como nuevo representante en la RPCh. La cuestión de la normalización ha sido, incorrectamente, señalada como el momento en el cual ambos países establecen relaciones diplomáticas y eso conlleva a un dato inexacto.

La segunda etapa de las relaciones chino-argentinas difiere de la primera en la importancia política y estratégica que va cobrando China en la política internacional y, posteriormente, en la economía mundial. En esta segunda etapa se verifica una profundización de los lazos cooperativos e incremento del intercambio comercial entre ambos países. No obstante, cabe destacar que la semejanza entre ambos segmentos temporales está dada en que las relaciones se desarrollaron entre Estados periféricos o semi-periféricos (ninguno de los dos podía considerarse como un país central).

Entre 1997 y 1999, China cierra el periodo colonial de su historia por la recuperación de los territorios de Hong Kong y Macao, respectivamente. Más aún, desde 1999 China es incorporada al oligopolio económico mundial (Bolinaga, 2013b, p. 197). En diciembre de 2001, adhiere a la Organización Mundial de Comercio (OMC) y eso reafirma su integración a la economía mundial y ya desde 2009 se posicionó como la segunda eco- 
nomía mundial. Todo esto, sumado a los otros indicadores que se vienen desarrollando, hace que China ya no sea una potencia emergente sino una potencia emergida, es decir, ya no es un país semi-periférico sino un país central. El gran legado de Deng Xiaoping es una China integrada a la economía mundial: "Únicamente quienes vivieron en la China de Mao Zedong pueden valorar en toda su extensión las transformaciones llevadas adelante por Deng Xiaoping." (Kissinger, 2012, p. 336).

La modernización económica en China contribuyó al tránsito del epicentro económico hacia el Pacífico Norte y eso condicionó la orientación de las políticas exteriores y del flujo comercial del resto de los países del mundo. En el caso concreto de Argentina, el ascenso chino profundizó progresivamente una asimetría política y comercial. Es decir, a diferencia de lo acontecido durante las primeras dos etapas (1945/1972 y 1972/2004) donde las relaciones se desarrollan entre Estados periféricos o semi-periféricos; en la tercera etapa (2004/2014) el vínculo se reformuló en términos de centro-periferia. Se tomó particularmente el 2004 como inicio de esta última etapa porque es cuando se comienza a verificar mayor influencia de China en la región de América Latina y el Caribe y porque marca el inicio de una "asociación estratégica" (Bolinaga, 2013a). De tal forma, el criterio político de asimetría supone una China devenida en gran potencia (país central) y una Argentina que continúa como potencia media (país periférico/semi-periférico), según su posicionamiento en la estructura internacional de poder y su incidencia en la economía mundial. Por su parte, el criterio comercial de asimetría operó sobre dos niveles: a) reproduce el tradicional esquema de intercambio comercial centro-periferia (manufacturas por materias primas) y b) el peso relativo de uno y otro país como socio comercial.

La tercera etapa coincide con el momento en el cual China terminó de configurarse como un socio estratégico de Argentina. A diferencia de lo que aconteció con Estados Unidos, las relaciones bilaterales siempre han sido cordiales y de amistad. Más aún, las estructuras productivas son complementarias, sobre todo a la luz del proceso de modernización e industrialización en China, y eso generó un mayor dinamismo comercial en el intercambio bilateral (ver Tabla 5). 
Tabla 5

Comercio sino-argentino (1972-2014)

\begin{tabular}{|c|c|c|c|}
\hline Año & Exportaciones & Importaciones & Saldo \\
\hline 1972 & 2.500 & 426 & 2.074 \\
\hline 1973 & 16.280 & 1.700 & 14.580 \\
\hline 1974 & 90.000 & 1.000 & 89.000 \\
\hline 1975 & 20.000 & 1.000 & 19.000 \\
\hline 1976 & 2.752 & 242 & 2.510 \\
\hline 1977 & 86.957 & 625 & 86.332 \\
\hline 1978 & 64.508 & 2.424 & 62.084 \\
\hline 1979 & 193.000 & 11.668 & 181.332 \\
\hline 1980 & 188.792 & 32.270 & 156.522 \\
\hline 1981 & 92.151 & 21.378 & 70.773 \\
\hline 1982 & 136.596 & 10.219 & 126.377 \\
\hline 1983 & 498.641 & 4.918 & 493.723 \\
\hline 1984 & 74.851 & 6.075 & 68.776 \\
\hline 1985 & 311.004 & 4.340 & 306.664 \\
\hline 1986 & 252.053 & 9.607 & 242.446 \\
\hline 1987 & 265.575 & 12.765 & 252.810 \\
\hline 1988 & 361.650 & 10.782 & 350.868 \\
\hline 1989 & 407.204 & 15.116 & 392.088 \\
\hline 1990 & 240.970 & 12.111 & 228.859 \\
\hline 1991 & 247.513 & 188.200 & 59.313 \\
\hline 1992 & 128.296 & 483.890 & -355.594 \\
\hline 1993 & 163.250 & 626.100 & -462.850 \\
\hline 1994 & 268.500 & 728.800 & -460.300 \\
\hline 1995 & 285.741 & 607.891 & - 322.391 \\
\hline 1996 & 607.446 & 697.888 & -90.442 \\
\hline 1997 & 871.009 & 1.005 .580 & -134.571 \\
\hline 1998 & 681.788 & 1.167 .498 & -485.710 \\
\hline 1999 & 507.902 & 992.123 & -484.221 \\
\hline 2000 & 769.224 & 1.156 .738 & -360.514 \\
\hline 2001 & 1.109 .158 & 1.065 .269 & 43.889 \\
\hline 2002 & 1.098 .558 & 330.240 & 768.318 \\
\hline 2003 & 2.452 .939 & 720.240 & 1.732 .699 \\
\hline 2004 & 2.628 .323 & 1.401 .814 & 1.226 .509 \\
\hline 2005 & 3.158 .285 & 2.239 .552 & 918.732 \\
\hline 2006 & 3.506 .134 & 3.121 .889 & 384.245 \\
\hline 2007 & 5.166 .609 & 5.092 .949 & 73.660 \\
\hline
\end{tabular}




\begin{tabular}{lccc}
\hline & & & \\
2008 & 6.397 .528 & 7.104 .380 & -706.853 \\
2009 & 3.668 .283 & 4.822 .595 & -1.154 .312 \\
2010 & 5.858 .682 & 7.648 .853 & -1.790 .171 \\
2011 & 6.176 .281 & 10.573 .351 & -4.397 .069 \\
2012 & 5.165 .455 & 9.954 .270 & -4.788 .815 \\
2013 & 5.762 .377 & 11.312 .451 & -5.550 .074 \\
2014 & 4.650 .042 & 10.761 .477 & -6.111 .434 \\
\hline
\end{tabular}

Fuente: Elaboración propia en base a estadísticas del INDEC (1972/2000; 2001/2014).

Según los datos de la tabla 5, entre 1972 y 1991, el saldo comercial fue constantemente positivo para Argentina. Recién con la liberalización del comercio y con la política de tipo de cambio fijo se deterioran los términos del intercambio: entre 1992 y 2000 hubo un déficit comercial creciente. Desde 2001 el intercambio volvió a ser positivo para Argentina pero no duro demasiado, ya en 2008 volvió a emerger un saldo negativo para el país.

Profundicemos. La negociación e implementación de convenios comerciales alcanzados bajo el gobierno militar, permitió alcanzar picos comerciales sin precedente en la relación comercial bilateral pero durante el gobierno de Alfonsín el intercambio se estancó. Lo cual no niega que sí se hayan alcanzado varios acuerdos importantes en otras áreas, por ejemplo en materia de sanidad y cuarentena animal y vegetal. Más aún, China ya era tan importante que aún en el contexto de una política de defensa de los derechos humanos y del proceso de redemocratización, ni el gobierno de Alfonsín ni el de Menem condenaron los acontecimientos de la Plaza de Tiananmen en 1989.

La importancia que otorgó el gobierno de Menem a la RPCh, en su modelo de vinculación con el mundo, se explica por la mayor relevancia política y económica de este actor en el sistema internacional y en la economía mundial. De hecho, no puede ignorarse que el mismo presidente consideró a China como la segunda economía mundial (Menem, 1999, p. 197). En concreto, la orientación política hacia China se insertó en un marco estratégico que tuvo por objeto reposicionar al país en el Pacífico Norte, principalmente, con las dos potencias de la región: Estados Unidos y China.

Desde los años 90, China reduce su exportación de minerales, petróleo y alimentos abruptamente y se convierte en un importador neto, dada la insuficiencia de su oferta interna en el contexto de la modernización económica por la que atravesaba. Desde prin- 
cipios del siglo XXI la demanda china de materias primas mejoró el nivel de precios de los commodities y a esto también se sumó la salida de la convertibilidad que, por traumática que fuera, estimuló las exportaciones argentinas hacia el mercado chino. Así, desde 2001, se invierte la balanza comercial a favor de Argentina quien pasa a registrar importantes superávits comerciales. Sin embargo, el ritmo de crecimiento de las exportaciones se desaceleró desde 2004, al tiempo que se aceleró la dinámica de las importaciones: las ventas argentinas a China en 2008 crecieron 160\% respecto de 2003 pero las compras lo hicieron en $886 \%$. Esta tendencia se agudizó más aún desde entonces, con lo cual para 2014 las exportaciones crecieron casi 90\% respecto de 2003 pero las importaciones más del $1.300 \%$. De modo que, según la tabla 5 , el superávit se fue achicando sistemáticamente y, entre 2008 y 2014, Argentina volvió a quedar presa de un déficit estructural con China: se acumuló una pérdida por más de 25.000 millones de dólares. En suma, el "factor chino" es clave para comprender la fuga de divisas que pesó sobre el país en ese periodo.

La gran paradoja es que en noviembre de 2004, los presidentes Néstor Kirchner y Hu Jintao habían firmado en Buenos Aires un memorándum de entendimiento, en el cual se fijaban tres elementos centrales: a) la formación de una "asociación estratégica"; b) Argentina reconoce el estatus de "economía de mercado" a China (Artículo 1) y c) se incrementarían las ventas argentinas en al menos 4.000 millones de dólares por sobre el nivel registrado en ese entonces (Artículo 2). Así, salen a la luz tres factores a considerar:

Primero: se cambió una posición concreta en el presente (el reconocimiento como economía de mercado) por una promesa a futuro anclada en un océano de incertidumbres.

Segundo: el incremento de los 4.000 millones de dólares se alcanzó aproximadamente hacia el año 2008, no obstante, desde entonces las exportaciones se estancaron y decrecieron.

Tercero: la ampliación del déficit comercial estructural a favor de China.

¿Qué significó otorgar el estatus de "economía de mercado"? China había sido tipificada por la OMC como una economía en transición, así la comparación de precios podía realizarse por medio de terceros países. Argentina cercenó su margen de acción al tiempo que también desconoció la decisión de la OMC de reconocer a China como economía en transición. Entonces, el cálculo final ha sido -en su gran mayoría- más elevado que el que hubiera resultado de utilizar los precios y costos vigentes en China, esto comprometió a los industriales argentinos. Así, el bilateralismo profundizado por la praxis 
política china conllevó a una mayor asimetría de poder que la que se hubiera verificado en el plano multilateral. Aun cuando la retórica oficial china promueve un "sistema multilateral de comercio" su praxis circula en sentido contrario.

También hay que considerar que China ha sido receptora aproximadamente del $20 \%$ de las medidas antidumping aplicadas en el mundo (Tussie y Bianchi, 2004). Pero entonces: ¿por qué Argentina otorgó este reconocimiento? La asimetría de poder a favor de China o la decisión de Brasil de reconocerla como tal y, en consecuencia, la reducción del margen de acción del Palacio San Martín fueron factores condicionantes del accionar argentino. Pero, indudablemente, el argumento de mayor peso es que China -siendo el segundo socio comercial de Argentina- podría haber afectado la inserción comercial internacional del país.

El gobierno de Beijing podría haber adoptado medidas retorsivas que conllevaran a una caída del comercio bilateral, por ejemplo por medio de medidas de tipo fitosanitarias sobre granos o similares al llamado "Aviso 73" (emitido en 2004, implicó la fijación unilateral de un estándar nacional superior al internacional en lo que respecta al aceite de soja). Otro ejemplo más reciente tuvo lugar cuando Beijing implementó la norma BT 1535/2003 para establecer un límite máximo de 100 partes por millón de residuos de hexano para los embarques de aceite crudo de soja y generó alerta en torno al comercio bilateral. No es casual que esto sucediera cuando la venta de aceite de soja superó a las exportaciones de poroto de soja. China protege su propia capacidad de molienda y eso va poniendo al descubierto como condiciona la capacidad argentina para incorporar valor agregado a sus exportaciones. Mientras que en 2009, el aceite de soja representó el 39\% y el poroto de soja 33\% del total exportado por Argentina a China, para el año 2010 la relación se reformuló en 3\% y 71\%, respectivamente según datos obtenidos de COMTRADE.

Al tomar posición sobre los tres factores mencionados ut supra cabe preguntarse: ¿hay una asociación estratégica o una asociación asimétrica entre Argentina y China? La conclusión a la que se arribó se centra en dos conceptos: a) la reformulación del esquema de vinculación en términos de centro-periferia y b) la reprimarización productiva del comercio sino-argentino. Ambos conceptos se nutren en el peso relativo de uno y otro país en el esquema global comercial de cada uno (Tabla 6) y un análisis de la composición sectorial de las exportaciones argentinas al mercado chino (Tabla 7 y Figura 1).

La tabla 6 muestra la asimetría de China y Argentina como socios comerciales. Argentina pasó de ocupar el puesto $23^{\circ}$ en 1990 como proveedor de China para caer sistemáticamente al puesto $41^{\circ}$ en 2013. Por el contrario, en materia de exportaciones chi- 
nas Argentina pasó de ser el destino número $86^{\circ}$ al puesto $6^{\circ}$, en iguales periodos. Cabe destacar que en ambas variables, el volumen total de intercambio está por debajo del $1 \%$ del total comercializado por China. En contraste, el peso de China como socio argentino es significativamente mucho más relevante. En materia de exportaciones, China pasó del puesto $14^{\circ}$ en 1990 a ocupar el $2^{\circ}$, ya en 2013; mientras que en las importaciones escaló de la posición $22^{\circ}$ al $2^{\circ}$ puesto, en igual periodo de tiempo. Además, la RPCh representó poco más del $7 \%$ y del 15\%, respectivamente, de las exportaciones e importaciones totales de Argentina en 2013. La asimetría es clara y eso también contribuye a explicar la reducción del margen de negociación para el Palacio San Martín en la última década.

Tabla 6

China y Argentina socios asimétricos

\begin{tabular}{ccccc}
\cline { 2 - 5 } & \multicolumn{3}{c}{ Participación y posición de Argentina en el comercio chino } \\
\cline { 2 - 5 } Año & $\begin{array}{c}\text { Participación de } \\
\text { Argentina }\end{array}$ & $\begin{array}{c}\text { Posición de } \\
\text { Argentina }\end{array}$ & $\begin{array}{c}\text { Participación de } \\
\text { Argentina }\end{array}$ & Posición de Argentina \\
\hline 1990 & $0,60 \%$ & 23 & $0,02 \%$ & 86 \\
2013 & $0,31 \%$ & 41 & $0,40 \%$ & 36
\end{tabular}

\begin{tabular}{ccccc}
\cline { 2 - 5 } & \multicolumn{3}{c}{ Participación y posición de China en el comercio argentino } \\
\cline { 2 - 5 } Año & $\begin{array}{c}\text { Participación de } \\
\text { China }\end{array}$ & Posición de China & $\begin{array}{c}\text { Participación de } \\
\text { China }\end{array}$ & Posición de China \\
\hline 1990 & $1,95 \%$ & 14 & $0,78 \%$ & 22 \\
2013 & $7,19 \%$ & 2 & $15,34 \%$ & 2 \\
\hline
\end{tabular}

Fuente: Bolinaga y Slipak (2014).

Las cifras registradas en la tabla 7 explican la alta concentración de las exportaciones en el complejo oleaginoso y el proceso de reprimarización del vínculo comercial, entre 2001 y 2013. En dicho periodo las exportaciones argentinas se concentraron en tres grandes rubros: poroto de soja, aceite de soja y crudo de petróleo. Mientras que en los dos primeros periodos (2001/04 y 2005/09) representaron el $78 \%$ y el $73 \%$ respectivamente, estos tres productos pasaron a representar el $90 \%$ del total exportado a China en el tercer 
periodo seleccionado (2010/13). Sólo el complejo oleaginoso represento el 79\% del total, en este último periodo. Entonces, el comercio dista de estar diversificado y, además, está claramente concentrado en productos primarios y sus derivados.

Tabla 7

Principales exportaciones argentinas a China, periodos seleccionados

\begin{tabular}{lcccccc}
\cline { 2 - 6 } & $2001 / 2004$ & $\%$ & $2005 / 2009$ & $\%$ & $2010 / 2013$ & $\%$ \\
\hline Poroto de soja & 3.713 .468 & 50 & 9.420 .765 & 43 & 16.125 .735 & 70 \\
Crudo de Petróleo & 180.835 & 2 & 2.185 .195 & 10 & 2.402 .135 & 11 \\
Aceite de Soja & 1.880 .112 & 26 & 4.311 .509 & 20 & 2.133 .937 & 9 \\
\hline Total 3 productos & 5.774 .415 & 78 & 15.917 .469 & 73 & 20.661 .807 & 90 \\
Total Productos Restantes & 1.549 .000 & 22 & 5.901 .000 & 27 & 1.901 .000 & 10 \\
Total & 13.097 .830 & 100 & 37.735 .938 & 100 & 43.224 .614 & 100 \\
\hline
\end{tabular}

Unidad Monetaria: miles de USD corrientes

Fuente: Elaboración propia con información de Trade Map.

Al matizar los subperiodos (ver Tabla 7) se puede corroborar que el peso de las exportaciones de poroto de soja se incrementó de $50 \%$ a casi un $70 \%$; mientras que la venta de aceite de soja cayó de $26 \%$ a tan solo un $9 \%$. Por tanto, la relación comercial se reprimarizó. Es decir, se reorientaron los factores productivos hacia actividades de menor agregación de valor. Lo que terminó determinando esta tendencia es que entre 2010 y 2013 el crudo de petróleo desplazó al aceite de soja como segundo producto más importante. Así, el producto con mayor grado de agregación de valor en 2013 cayó al tercer puesto: el poroto de soja representó el 58\%, el crudo de petróleo un 13\% y el aceite de soja un $10 \%$. 


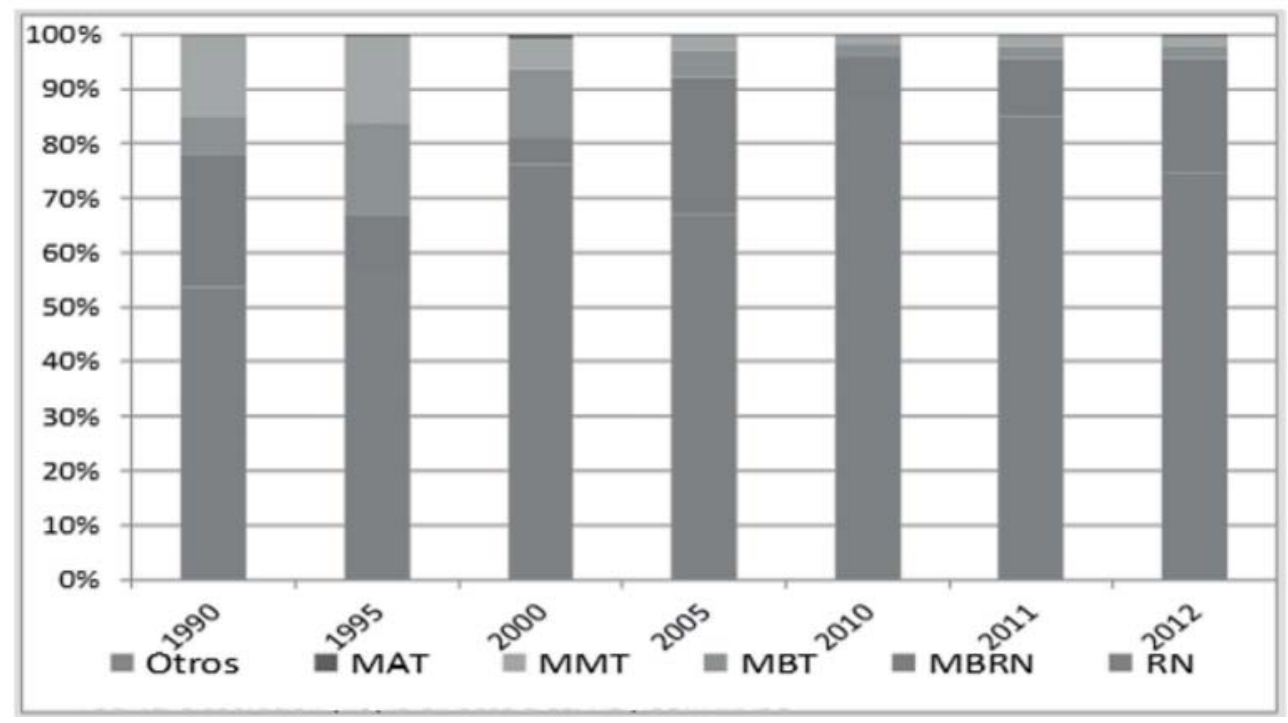

Figura 1. Exportaciones argentinas a China, por contenido tecnológico (1990-2012). MAT: Manufacturas de Alto Contenido Tecnológico / MMT: Manufacturas de Medio Contenido Tecnológico / MBT: Manufacturas de Bajo Contenido Tecnológico MBRN: Manufacturas basadas en Recursos Naturales / RN: Recursos Naturales. Fuente: Bolinaga y Slipak (2014).

En suma, no se logró diversificar la oferta exportadora anclada en productos primarios y tampoco se pudo incrementar el valor agregado de las manufacturas de origen agropecuario. La figura 1 muestra las exportaciones argentinas a China según contenidos tecnológicos y reafirma esta apreciación. Entre 2005 y 2012, la suma de recursos naturales y manufacturas basadas en recursos naturales superó en promedio el $90 \%$ del total exportado por Argentina a China. Mientras que en 1990 ambos rubros representaron el 78\% para el 2012 pasaron a manifestar el 96\%. En contraste, las manufacturas de bajo, medio y alto contenido tecnológico exportadas desde Argentina a China se contrajeron de 22\% a 4\%, en igual periodo de tiempo. El proceso de reprimarización productiva por el que atravesó Argentina en su esquema comercial con China es lo que limitó específicamente la posibilidad de sacar mayor provecho del intercambio comercial entre ambos países. 


\section{Conclusión}

Argentina no está aislada de la configuración de poder en el sistema internacional. A lo largo de su historia su inserción internacional estuvo condicionada -en mayor o menor medida- por los esquemas de vinculación con las grandes potencias. Los países periféricos se vinculan con las grandes potencias ya sea por afinidad, seguridad, comercio u otras razones. Pero dado que las unidades alteran su posición en la estructura, el ciclo de ascenso y descenso de grandes poderes condiciona la política comercial y exterior de los países restantes del sistema internacional, es decir, de las unidades periféricas.

China ha ganado progresivamente un papel sustancial en la política comercial y exterior argentina. Dicha coyuntura, se acelera en el segmento temporal que transcurre entre 2004 y nuestros días, como consecuencia de la incorporación de China al directorio de grandes potencias y de su rol en el nuevo eje económico mundial del Pacífico Norte. La diferencia con las otras etapas de la relación bilateral, es que en ésta se imprime claramente el vínculo centro-periferia y se deja de lado las relaciones entre Estados semi-periféricos.

El realismo periférico como teoría normativa de las relaciones internacionales favorece el incremento de vinculación con los centros de poder pero al mismo tiempo limita esa vinculación en tanto sostiene que la única confrontación que debe mantenerse con las grandes potencias es aquella que sea necesaria para no empeñar el desarrollo económico propio. Es decir, la política comercial y exterior se convierten en un instrumento al servicio de un modelo de desarrollo económico. En el caso argentino, primó más el vínculo con la gran potencia que el modelo de desarrollo en sí mismo. Frente al nuevo tránsito del epicentro económico y el ascenso de China, indudablemente, Argentina vuelve a enfrentar el desafío de adaptarse a los cambios sistémicos. Pero también es cierto que tiene la posibilidad de mejorar su posicionamiento internacional por medio de una política de Estado que miré hacia el Pacífico Norte y, específicamente, hacia China.

No obstante, no alcanza con tener una política reactiva como la que se ha tenido hasta ahora, sino que es necesario pensar en un modelo de desarrollo nacional que se instrumente por medio de políticas comerciales y de una política exterior basada en el realismo periférico puro. Es decir, el límite para confrontar con las grandes potencias siempre fue y será la defensa del desarrollo económico nacional. Entonces, frente a la reprimarización productiva, el déficit estructural y la creciente extracción de crudo de petróleo, Argentina debe confrontar con China en pos de mantener un vínculo de cooperación pero no de subordinación que condicione 
su desarrollo económico y facilite un nuevo capítulo de vaciamiento y empobrecimiento. Ese es el gran desafío que tiene la República Argentina por delante. Y, al mismo tiempo, el gran desafío de toda la región ante lo que ya se perfila como el Consenso de Beijing.

\section{Referencias}

Arce, J. (1958). Mi vida. Buenos Aires, Argentina: La Imprenta Científica.

Bolinaga, L. (2011). Estudio de los cambios en la orientación externa de un país periférico: ascenso de China, cambio en el epicentro económico hacia el Pacífico Norte y política exterior argentina (1989-2007). Tesis para optar al grado de doctor, Universidad Nacional de Rosario, Facultad de Ciencia Política y Relaciones Internacionales, Argentina.

- - - (2013a). ¿Apuesta China a la modernización productiva de América Latina? Realidad Económica, 279, 132-147.

- - - (2013b). China y el epicentro económico del Pacífico Norte. Buenos Aires, Argentina: Editorial Teseo.

Bolinaga, L. y Slipak, A. (23 a 25 de julio de 2014). Límites teóricos y fácticos a la categoría de cooperación sur-sur» para analizar la vinculación entre China y Argentina (19892014). Ponencia presentada en la Joint International Conference ISA-FLACSO (Global and Regional Powers in a Changing World), Bueno Aires, Argentina.

Bregolat, E. (2011). La segunda revolución China. Claves para entender al país más importante de siglo XXI. Buenos Aires, Argentina: Capital Intelectual.

Burgin, M. (1969). Aspectos económicos del federalismo argentino. Buenos Aires, Argentina: Solar-Hachette.

Centro de Comercio Internacional (CIT). Análisis e investigación de mercados, Centro de Comercio Internacional, TRADE MAP. Recuperado de http://www.trademap.org/Index.aspx

Comisión Económica para América Latina y el Caribe CEPAL (2015). América Latina y el Caribe y China. Hacia una nueva era de cooperación económica. Santiago, Chile: Naciones Unidas. 
-—- Base de Datos y Estadísticas de la Comisión Económica para América Latina y Caribe. Disponible en http://www.trademap.org

Escudé, C. (1983). La Argentina Vs las grandes potencias (El precio del desafío). Buenos Aires, Argentina: Editorial de Belgrano.

- - . (1995). El realismo de los Estados Débiles. La política exterior del primer gobierno de Menem frente la teoría de las relaciones internacionales. Buenos Aires, Argentina: Grupo Editor Latinoamericano.

- - - (26 de julio de 2011). China, una oportunidad histórica. Diario La Nación. Buenos Aires, Argentina.

-_- (2012). Principios de Realismo Periférico. Una teoría argentina y su vigencia ante el ascenso de China. Buenos Aires, Argentina: Lumiere.

Escudé, C. y Cisneros, A. (2000). Historia general de las relaciones exteriores de la República Argentina. Buenos Aires, Argentina: Grupo Editor Latinoamericano. Recuperado de http://www.argentina-rree.com/home_nueva.htm

Gosende, E. (2007). Modelos de politica exterior argentina: alternativas para salir del modelo conservador-menemista. Buenos Aires, Argentina: Libros de Tierra Firme.

Instituto Nacional de Estadística y Censos (1964-2014). Cuadernos de Comercio Exterior. Buenos Aires, Argentina: INDEC.

- - . (2000). Indec Informa. Buenos Aires, Argentina: Ministerio de Economía y Producción, Instituto Nacional de Estadística y Censos.

- - - (2014). Indec Informa. Buenos Aires, Argentina: Ministerio de Economía y Producción, Instituto Nacional de Estadística y Censos.

Keohane, R. (2005). After hegemony. Cooperation and discord in the world political economy. New Jersey, Estados Unidos: Princeton University Press.

Kissinger, H. (2012). China. Buenos Aires, Argentina: Debate.

Lanús, A. (2000). De Chapultepec al Beagle. Política Exterior Argentina 1945-1980. Buenos Aires, Argentina: Emecé. 
Menem, C. (1999). Universos de mí tiempo. Buenos Aires, Argentina: Editorial Sudamericana.

Morgenfeld, L. (2011). Vecinos en conflicto. Argentina y Estados Unidos en las conferencias Panamericanas (1880-1955). Buenos Aires, Argentina: Ediciones Continente.

Naciones Unidas. Base de Datos Estadísticos de Naciones Unidas COMTRADE. Recuperado de http://comtrade.un.org/

Oviedo, E. (2005). China en expansión. Volumen 8. Córdoba, Argentina: Universidad Católica de Córdoba, Colección Thesys.

- - - (2010). Historia de las relaciones internacionales entre Argentina y China 1945/2010. Buenos Aires, Argentina: Editorial Dunken.

Puig, J. (Comp). (1984). América Latina: políticas exteriores comparadas. Volumen 1. Buenos Aires, Argentina: GEL.

Rapoport, M. (2000). Historia Económica, política y social de la Argentina. Buenos Aires, Argentina: Ediciones Macchi.

Renouvin, P. y Duroselle, J. (2001). Introducción a la historia de las relaciones internacionales. México: Fondo de Cultura Económica.

Svampa, M. (2013). Consenso de los Commodities y lenguajes de valoración en América Latina. Revista Nueva Sociedad, 244, 30-46.

Slipak, A. (29, 30 y 31 de agosto de 2013). ¿De qué hablamos cuando hablamos de reprimarización? Un aporte al debate sobre la discusión del modelo de desarrollo. VI Jornadas de Economía Crítica. Facultad de Ciencias Económicas de la Universidad Nacional de Cuyo, Mendoza.

- - - (5 y 6 de noviembre de 2014). La expansión de China en América Latina: incidencia en los vínculos comerciales argentino-brasileros. Congreso de Economía Política Internacional, Universidad Nacional de Moreno (UNM). Moreno (Buenos Aires). 
Tussi, D. y Bianchi, E. (2004). El reconocimiento de China como economía de mercado, LATN (occational paper). Recuperado del sitio Banco Interamericano de Desarrollo: http://www.iadb.org/intal/intalcdi/PE/2007/00883.pdf

Vázquez Presedo, V. (1992). Auge y decadencia de la economía argentina desde 1776. Buenos Aires, Argentina: ANCE. 